\title{
The Large Hadron Collider and the Role of Superconductivity in One of the Largest Scientific Enterprises
}

\author{
Lucio Rossi, Member, IEEE
}

\begin{abstract}
After ten years of R\&D and industrialization and seven years of construction, the LHC is near completion. The manufacture of the $\mathbf{1 7 5 0}$ main superconducting magnets and of the 8000 superconducting correctors for the accelerator, as well as their cold test at CERN, is approaching the end, while their commissioning in the $27 \mathrm{~km}$-long tunnel has started. The very large superconducting magnets for the main detectors, ATLAS and CMS, are installed and their commissioning is under way. Superconductivity is the key technology for the largest scientific enterprise of this decade: it accounts for half of the total cost and has proved to be affordable and reliable. Thanks to superconductivity we can probe new states of matters and reproduce conditions of 1 ps after the big bang. The paper will give the link between the Physics requirements and the answers that applied superconductivity has offered in this project.
\end{abstract}

Index Terms-Accelerators, accelerators and detector magnets, large-scale superconductivity, LHC, Nb-Ti superconductors.

\section{INTRODUCTION}

A CCELERATORS and superconductivity (SC) have been good companions for many years [1]-[5]. Starting from the Argonne bubble chamber [6], [7] which has been the first large superconducting magnet to be operated for Physics experiments and from the Tevatron [8], [9] which has been the first large accelerator based on superconductivity, High Energy Physics (HEP) has given a tremendous push to practical development of superconductivity. In particular, when the possibility of practical and economical application of SC was fading for the power sector, in the 1980s, HEP nurtured improvements as there was a series of projects requesting a continuous R\&D.

The Large Hadron Collider [10], near completion at CERN, is the last of a series of large size accelerators based on SC technology, and it has a size four times and field level more than twice that of previous HEP accelerators [9], [11], [12]. Also its main detectors, named ATLAS [13] and CMS [14] are based on SC magnets of size and energy never before attained. In total at the LHC some 15 GJ of magnetic energy will be stored in superconducting magnets. LHC will also be the first large scale application of HTS in an operating large device, by using 1180 currents leads, all based on $\mathrm{Bi}-2223$, of various amperage, up to $13 \mathrm{kA}$.

Manuscript received September 11, 2006.

The author is with CERN, Accelerator Technology Department, CERN-AT, Geneva 23, CH 1211 Switzerland (e-mail: lucio.rossi@cern.ch).

Color versions of one or more of the figures in this paper are available online at http://ieeexplore.ieee.org.

Digital Object Identifier 10.1109/TASC.2007.899260

\section{GOALS AND NEEDS OF HEP}

\section{A. Particle Physics and Accelerators}

Accelerators are one of the two frontiers of Physics. On one hand, astrophysics looks into giant objects like galaxies at farthest distances, $10^{25} \mathrm{~m}$, by means of optical and radio-telescopes. On the other hand, HEP looks into infinitesimally small dimensions, seeking the elementary constituents of our world and the forces that keep them together. In this respect accelerators are very powerful microscopes that can probe matter in very small detail. Indeed accelerators use particles at such energy that the associated wavelength $\lambda=\mathrm{h} / \mathrm{p}$, is very short, allowing details of the same order of magnitude to be resolved. In the $\mathrm{LHC}$, where elementary collisions will happen at the TeV scale, the corresponding $\lambda$ is $10^{-18} \mathrm{~m}$ : so we could rename our machines "attoscopes."

By reaching the energy that is necessary to probe matter in such detail, we also recreate conditions that existed only at the beginning of our world. The relationship between time after the big bang and the energy necessary to access it is given by: $\mathrm{t}=$ $1 / \mathrm{E}^{2}$, where $\mathrm{t}$ is in seconds and $\mathrm{E}$ is in $\mathrm{GeV}$. At the $\mathrm{TeV}$ scale reached in the $\mathrm{LHC}$, we will recreate conditions that existed 1 ps after the big bang: accelerators are real time machines, too.

HEP in the last 30 years has been able to give a fairly good description of the fundamental constituents, all condensed in the so-called Standard Model (SM). However one particle, absolutely needed for the consistency of the model, still escapes experimental detection: the Higgs particle. Moreover, although SM is a very detailed description, a few fundamental questions need still to be answered: i) why so many particles; ii) why so many forces; iii) what is mass, and why do particles have the masses they do ? LHC should give a decisive contribution to the understanding and solution of these enigmas and should also provide information and direction about other important questions, like the nature of dark matter and dark energy and the scale of grand-unification.

\section{B. Why Do We Need Technology "At the Edge”?}

1) The Energy Frontier: There are two routes to open new knowledge in particle physics, as illustrated by Fig. 1. One route is to access the highest energies, the so-called "energy frontier." In fact, by giving more and more energy to particles we can create new particles with rest mass equal to the kinetic energy of the accelerated particles, thus transforming energy into mass that is equivalent to looking deeper and deeper into sub-nuclear details, as mentioned above. 


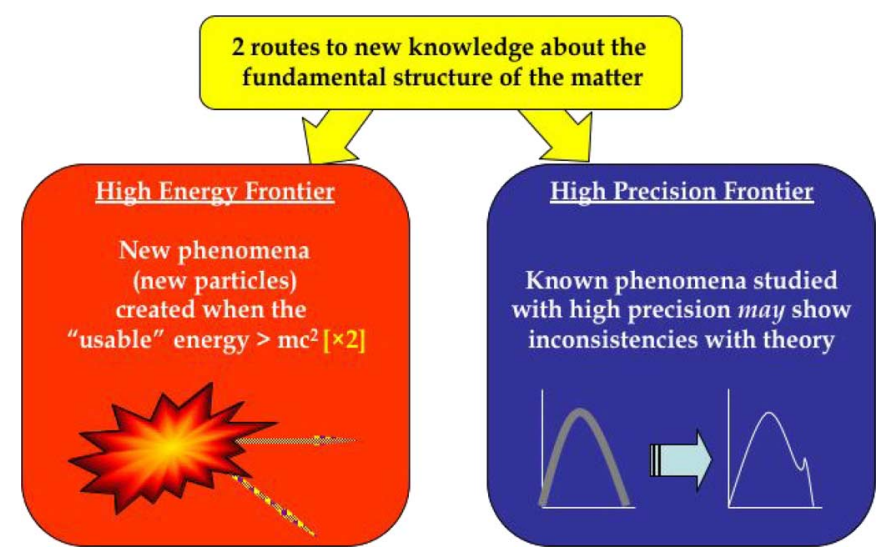

Fig. 1. The two routes of HEP.

For the high-energy frontier, like the LHC, the accelerated particles are hadrons (mostly protons, sometimes ions) since for them the power lost by synchrotron radiation is small and therefore it is convenient to re-circulate the beam through short sections of radio-frequency cavities providing the accelerating electric field. Usually two counter-rotating beams are collided continuously at a few points of the ring. Hadron colliders are thus circular and based on bending magnets.

The energy of particle delivered by circular accelerators in relativistic regime can be written as [5]: $\mathrm{E} \approx 0.3 \mathrm{~B} \mathrm{R}$, where the $\mathrm{E}$ is expressed in $\mathrm{TeV}, \mathrm{B}$ in Tesla and $\mathrm{R}$ in $\mathrm{km}$. One can see that high field pays off as much as large tunnel size hence is clear the interest and the push toward high field magnets. Since the end of the 1970s all large hadron colliders built, or conceived, are based on superconductivity.

There are also stringent economical reasons that make accelerators a so-called "killer application," i.e., where superconductivity is indispensable. The power dissipated in the resistive coils of normal conducting magnets scales as BR, i.e. as the beam energy, while the cryogenic power needed for the superconducting magnet scales to first order as R only, which makes evident the advantage of choosing high field and "moderate" $\mathrm{R}$ in selecting the parameters of the collider. In reality the previous scaling is only approximate since cryogenic power is affected significantly also by the operating temperature $(1.9 \mathrm{~K}$ in the $\mathrm{LHC}$ rather than the $4.4 \mathrm{~K}$ used in other machines) and, for machines beyond the LHC energy, also by the synchrotron radiation power. LHC absorbs about $40 \mathrm{MW}$ of electric power for the cryogenic plant for the super-conducting magnets of $8.3 \mathrm{~T}$ installed in the $27 \mathrm{~km}$ tunnel: if normal conducting magnets operating at $1.8 \mathrm{~T}$ were used, a $100 \mathrm{~km}$ long tunnel would have been required with an electrical power consumption of $900 \mathrm{MW}$, leading to prohibitive capital and operation cost [15].

2) High Precision Frontier: The second route is the "high precision frontier", where by means of leptons (usually e $+\mathrm{e}-$ ) one can unveil information that can prove or disprove a theory. Although the energy is usually less than in hadron colliders, thanks to the cleanness of the particle-antiparticle annihilation, previous curves can reveal details that can confirm or invalidate the Standard Model, see Fig. 1. This route is pursued by large electron linacs, like the proposed ILC [16] requiring a massive

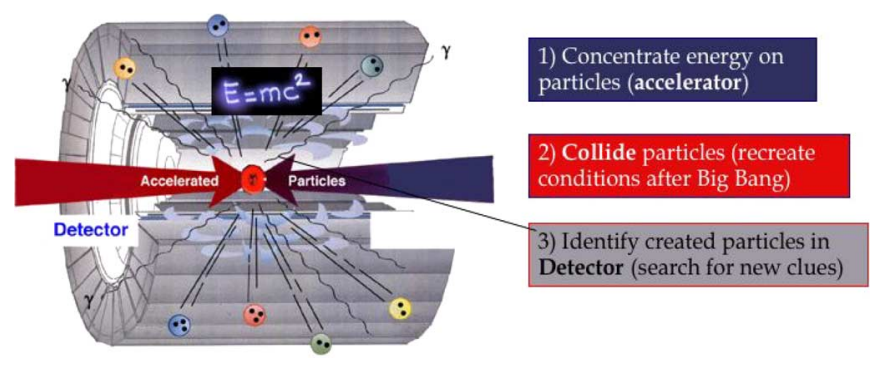

Fig. 2. Function of a particle and radiation detector.

use of RF. cavities working in the range 0.1 to $3 \mathrm{GHz}$. In this case the beam energy is expressed as: $\mathrm{E}=e \varepsilon \mathrm{L}$, where $\mathrm{L}$ is the length of the accelerating cavities providing electric field $\varepsilon$ and $e$ is the electron charge. In the case of linacs the comparison between superconducting and normal cavities is subtler. The highest gradients are actually reached with very high frequency copper cavities. However, the higher power dissipation, the smaller beam tube and the shorter bunches connected to use of high frequency copper cavities makes superconductivity a much better choice, at least in the range of energy today attainable, 0.5 to $1 \mathrm{TeV}$ in the center-of-mass. For these reasons the next precision frontier machine, called International Linear Collider is being designed based on SC technologies.

\section{Particle Detectors}

In both cases, once the particle are accelerated and smashed one against the other, the new particles and radiation coming off the collision point needs to be detected. To this aim the collision points are hermetically covered by $4 \pi$ detectors, intercepting all collision products, except for a very tiny cone along the primary beam axis, see Fig. 2. Detectors need magnetic fields for charge determination and for momentum spectrometry, whose resolution scales as: $\Delta p / p \propto B L^{2}$, when tracking is inside the magnetic field. Hence large volume pays off more than high field. However, in many cases, the parameters are such that SC magnets are necessary to obtain the necessary resolution.

In some cases the magnet is inside the calorimetric part of the detector: in these cases the magnet must be as transparent as possible to particle and radiation. Even if the field level is usually less than 2 Tesla, this condition can be achieved only by use of superconducting coils.

\section{ThE LARGE HADRON COLlideR}

LHC is designed to provide collision at $14 \mathrm{TeV}$ in the center of mass with a beam current of $0.5 \mathrm{~A}$. To do so, protons at low energy are injected from the existing SPS accelerator at CERN into the LHC main ring. Meanwhile the field of the dipoles is raised in 20 minutes from $0.45 \mathrm{~T}$ up to $8.3 \mathrm{~T}$ and the energy of the two counter-circulating beams is increased from 0.4 to $7 \mathrm{TeV}$. The main dipoles, and all other main systems, are designed for possible ultimate operation at $9 \mathrm{~T}$. In order to attain such high field with well-proved $\mathrm{Nb}$-Ti based technology, the magnets are cooled to $1.9 \mathrm{~K}$ by means of pressurized superfluid helium. In total some 40000 tons of mass are cooled to this low temperature, well below the $\approx 3 \mathrm{~K}$ relic temperature of the universe. 


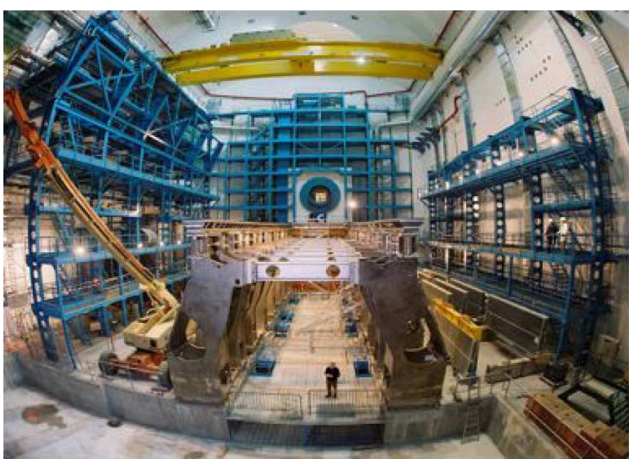

Fig. 3. The large ATLAS cavern in March 2004, ready to receive the ATLAS toroid. In the center, the tunnel of the LHC machine is also visible.

The LHC accelerator is complemented by four main experiments: ATLAS, CMS, Alice and LHC-b. The first two experiments are general-purpose experiments and the largest in size. Both use large superconducting magnets with a 1000 tons cold mass for each.

The $3.8 \mathrm{~m}$ diameter tunnel located some $100 \mathrm{~m}$ under ground, had been already built for the LEP project (an $\mathrm{e}^{+} \mathrm{e}^{-}$collider, which ceased to operate in 2000), while the ATLAS and CMS experiments are located in huge galleries, see Fig. 3.

The LHC project (the machine plus experimental areas) cost about 2.5 billion Euros (only material is accounted). It is worth noting that the cryo-magnetic system takes a most of it, with $2 / 3$ of the total budget allocated for it.

The project was approved in its final configuration in December 1996 and it is scheduled to be meet the milestone of the first beam circulating at injection energy by the end of 2007 . This is two years delay with respect to the original schedule fixed at the end of 1996 despite the budget difficulties encountered by CERN, the late starting of the magnet industrial production, and a few severe technical and organizational problems in the cryogenic distribution line, which runs all along the $27 \mathrm{~km}$ tunnel, and in the cryogenic/electrical distribution feed boxes.

\section{LHC MACHINE SuPERCONDUCTING MAGNETS}

\section{A. Generalities and Historical Background}

In iron-dominated magnets, the pole shape dictates field quality, while in superconducting magnets the conductor position dictates the precision of the field. In order to obtain the desired field level and to meet efficiency and cost targets, the required current density is huge, around $400 \mathrm{~A} / \mathrm{mm}^{2}$ [17]. For comparison the average $\mathrm{J}$ in high field solenoids is around $100 \mathrm{~A} / \mathrm{mm}^{2}$ and for large fusion and detector magnets it is around $30-50 \mathrm{~A} / \mathrm{mm}^{2}$. Electromagnetic forces are considerable and since the coil geometry is not self-supporting, (see Fig. 4) the containment of the conductor is critical both for stability (see later) and for field quality. The beam circulates 500 millions time before being exhausted. The field accuracy must be controlled for each single harmonic at the level of $10-100 \mathrm{ppm}$, in a region very near $(1 \mathrm{~cm})$ the coils.

Another main characteristic of the LHC is that each electrical circuit consists of many magnets supplied with current in se-

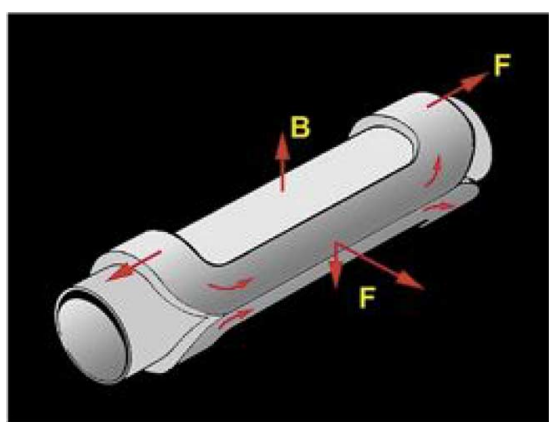

Fig. 4. Basic shape of a dipole coil.

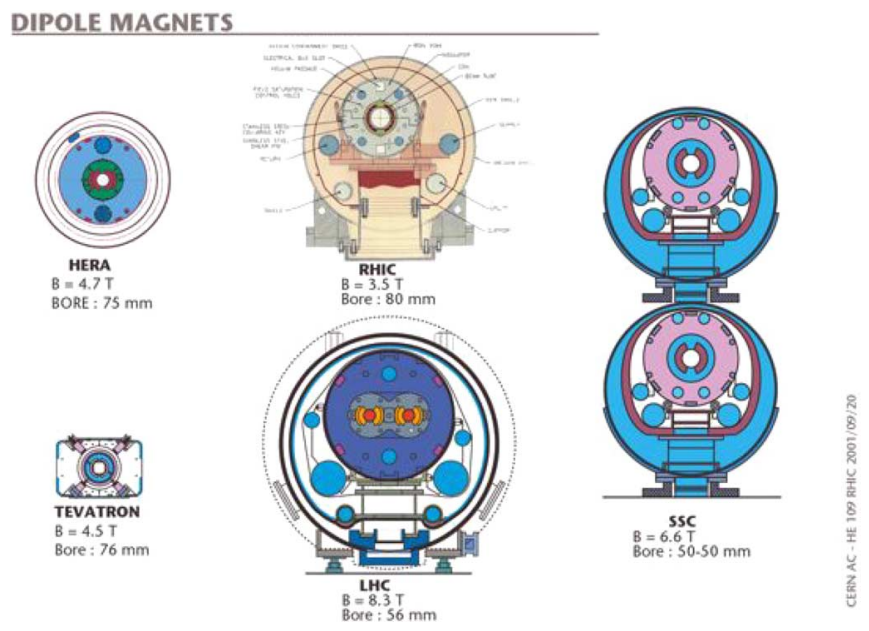

Fig. 5. The historical outlook of main HEP projects based on Sc magnets.

ries: There are $2 \times 154$ dipoles for each of the eight dipoles circuit, and there are 45 quadrupoles for each of the sixteen main quadrupole circuits. This implies that:

1) The magnets must be equal in bending (or focusing) strength to within $\sim 100 \mathrm{ppm}$. This was not an obviously attainable goal considering that LHC magnets were built in different production lines in different countries.

2) The worst magnet in quench performance will determine the final energy of the accelerator, without the possibility of compensating weak magnets with stronger ones.

Accelerator magnet operates near the critical surface: LHC dipoles operate at $85 \%$ of $\mathrm{I}_{\mathrm{C}}$ (measured on the load line) and can even operate at $93 \%$ (ultimate level of operation). This fact, together with the low content of stabilizer (typically $60 \%$ of the conductor cross section) implies that our magnets do train, which is the price to be paid for this very high field level when using $\mathrm{Nb}$-Ti. In Fig. 5 the cross sections of the principal hadron colliders main dipoles are shown. One can see that the cross sections look similar, but with two remarkable exceptions: i) the Tevatron, the pioneer, employed room temperature iron yoke, while all subsequent projects followed the line of HERA (derived from Isabelle, see Table 1) in having cold iron (less He consumption, more time to cool down and warm up); ii) the LHC has chosen a very compact design where the two beam channels are located in the same cold mass, following the Two-in-One concept. 


\section{B. The LHC Magnet}

1) Superconductors: All magnet coils in the LHC main ring (with the exception of a few magnets in special zones at high heat deposition by radiation) are wound from $\mathrm{Nb}$-Ti based conductor. Actually, at the very beginning of the LHC R\&D phase, a program based on $\mathrm{Nb}_{3} \mathrm{Sn}$ conductor working at $4.2 \mathrm{~K}$ was carried out as a possible alternative route to $\mathrm{Nb}$-Ti working at $1.9 \mathrm{~K}$ [18]. Despite some good technical success (the mirror coil was the first accelerator-like winding to break the $10 \mathrm{~T}$ threshold), the program was abandoned in 1991, in order to concentrate the limited available resources on the more practical and familiar $\mathrm{NbTi}$ route. However, in order to approach as much as possible the initial goal of $10 \mathrm{~T}$ field in the main dipoles [19], the decision was made to operate it at $1.9 \mathrm{~K}$ in a pressurized ( 1 bar absolute) superfluid helium bath. At $1.9 \mathrm{~K}$ the $\mathrm{Nb}$-Ti critical current curve is enhanced by $3 \mathrm{~T}$, with respect to the $4.2 \mathrm{~K}$ critical current. The actual gain in field obtained in a magnet is about $2 \mathrm{~T}$, thus permitting operation at $8.3 \mathrm{~T}$ and possibly at ultimate field of $9 \mathrm{~T}$. The maximum field estimated from measured short samples critical current is $9.65 \mathrm{~T}$.

For the LHC machine the total quantity of superconducting cable is $7600 \mathrm{~km}$ necessitating more than 400 tons of $\mathrm{Nb}-\mathrm{Ti}$ ingots of very high quality and homogeneity (at $0.1 \%$ level). To guarantee the necessary quality control many specific procedures have been implemented, from SPC (Statistical Production Control), to new tools to check automatically for cabling errors like strand crossovers, $\mathrm{Sn}-\mathrm{Pb}$ inclusions, sharp edges. An updated report on superconducting cables for the LHC is given in [20]

The critical current density of the whole cable production for the outer layer of the dipoles and for the quadrupole has exceeded the specification with a 5\%-10\% margin, little cabling degradation (2\%-3\%) and an excellent uniformity [21].

Magnetization is very important for accelerator magnets since it is the main source of field imperfections at injection energy. Much effort was made to control the magnetization, however a number of billets above specification had to be accepted. In a few cases the values were so high as to cause rejection of the billet. Large magnetization values have been traced to non-fully controlled conditions during extrusion. However through proper dilution of the high magnetization strands in cables dominated by low magnetization strands, it was possible to accept almost all of the production. This implied a remarkable effort of measurements at $1.9 \mathrm{~K}$ in the CERN superconducting laboratory. The quality assurance (QA) program for the LHC superconductor has been quite extensive but it has assured that all cable delivered to magnet manufactures, with the exception of one unit length of the early production, were free from significant defects.

The uniformity of interstrand resistance in the cable is important for controlling the field quality during the ramp up of the beam to flat top energy. The novel solution adopted for the LHC is based on coating the strands with a SnAg alloy, before cabling. After cabling, the cable roll is then exposed to a controlled heat treatment in air in order to oxidize the cables. This method is simple, cheap and suitable for Rutherford cabling techniques, but required many adjustments and practical $R \& D$ to become

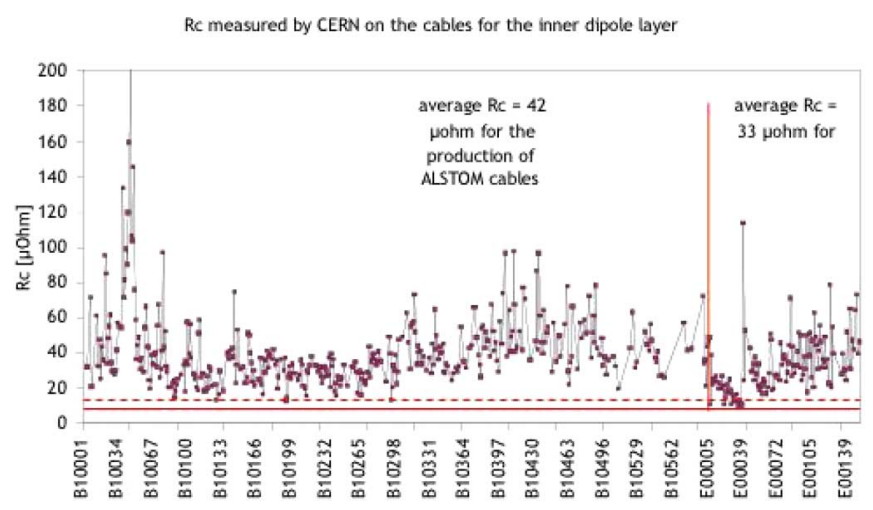

Fig. 6. Inter-strands contact resistance for the LHC cable 01, compared with target (dashed) and hard limit (solid horizontal line).

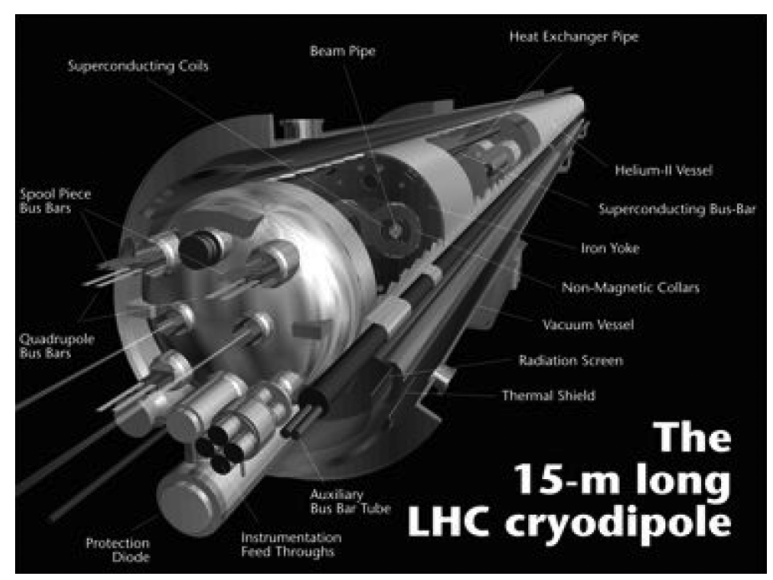

Fig. 7. Artistic view of the LHC main dipole.

reliable in industry. It has been certainly one of the keys to the success of the LHC cables (see Fig. 6).

2) Dipole Magnets (MB, Magnet Bend): The design of the LHC magnets went through about ten years of evolution with three generations of design. The three generations differ in the coil layout, in the collar design and on how the coil-collar assembly interferes with the yoke-skin assembly. The basic design characteristics [22] of the present third-final—generation are:

a) Collared coil: It is based on six conductor blocks. After an unsuccessful attempt to work with five coil blocks of the second generation, the third and final generation is based on an optimization where conductors are as radial as possible and shear forces are minimum. The design also allowed room for further optimizations, or cross-section fine-tuning, carried out twice during series construction, to better fit the beam dynamics targets [23]. The coils feature two layers, wound with two cables whose margins in critical current are very similar.

Due to the lack of stabilizer, to the very high current density and to the small operational margin, the quench energy is very small, ranging from tens of $\mu \mathrm{J}$ to $\mathrm{mJ}$. In such conditions, tiny movements of a few $\mu \mathrm{m}$ can trigger an irreversible transition. From this comes the great importance of the mechanical structure, and namely the collars that surround the coils (see Fig. 7).

Collars have been designed as Twin-type, a particular variant of the two-in-one where the two dipoles are coupled mechanically and also magnetically (no iron separation between the two 
coils. They are made of special austenitic steel with very low magnetization $\chi_{\mathrm{m}}<0.005$ under operating conditions [24]. They are obtained by fine blanking according to a shape that ensures the wanted coil cavity under stress and cold conditions. Austenitic steel allows a more comfortable margin in the construction and assembly tolerances, as shown in [25], with respect to aluminum. Also, thanks to its higher rigidity, the use of austenitic steel helps to limit conductor movements, an important issue in magnets where field accuracy is required at $1 \mathrm{~cm}$ from the conductor.

b) Iron yoke and cold mass assembly: Iron yoke: it not only serves for flux return, but it also adds about $15 \%$ to the field. An iron-free design would have only few percent less of central field, at the price of a much higher operating current, making more difficult operation and protection, in addition to the severe problem of high stray field.

Near the magnet ends, for $370 \mathrm{~mm}$, the laminations are composed of an outer shell of low carbon steel, like the main body, and with an inner shell, $20 \mathrm{~mm}$ thick of special austenitic steel. The latter is non magnetic and its mechanical properties, namely the thermal contraction, have been selected in order to fit exactly that of the iron. These nested laminations, are designed to lower the peak field on the coil end (always a quench risk region) while preserving the maximum magnetic length and reducing the quadrupole field component coming from aperture coupling at the end.

The magnet must be curved, with a sagitta of about $9 \mathrm{~mm}$, corresponding to a radius of curvature of $2812.36 \mathrm{~m}$. This curvature has a tolerance of $\pm 1 \mathrm{~mm}$, with the exception of the extremities of the magnet where the tolerance is very tight: $\pm 0.3 \mathrm{~mm}$ (systematic) and $0.5 \mathrm{~mm}$ r.m.s. in order to keep the corrector magnets centered with respect to the beam tube, to avoid harmonic feed down (detrimental for beam optics).

The outer shell, as mentioned above, is a shrinking cylinder, formed by welding two half-shells made out of $316 \mathrm{LN}$ stainless steel. The two shells have been produced curved with tolerances actually much worse than foreseen in the design. One remedy has been to couple shells with different bending radii such that a pair has average curvature inside a tolerable band. The two half-shells are welded onto the iron yoke in a huge and precise press with a given over-bend. Many trials were needed to finally control the spring back, which is not uniform among magnets. At the end the system of fixing the magnet inside the cryostat had to be changed after $10 \%$ of production and a sorting helped to place the magnet with sagitta out of tolerance in position of the ring less sensitive to such a deviation.

3) Quadrupole Magnets (MQ): Quadrupole magnets are necessary for focusing the beam and in the LHC they are of numerous types. Those classified as arc quadrupoles, MQ [22], [26], number 360. They are located in the Short Straight Section (SSS) cold mass, which comprises also different types of corrector magnets. Other quadrupoles are assembled in Special Short Straight Section cold masses (S4) and their total number is 32 for the dispersion suppressors (MQ-DS), 86 for the matching quadrupoles (MQM) and 24 for the wide aperture quadrupoles (MQY) [27]. Again, each S4 allocates one (or two in a few cases) quadrupole and a number of small and large correctors. Some MQM and all MQY are operated at $4.4 \mathrm{~K}$.

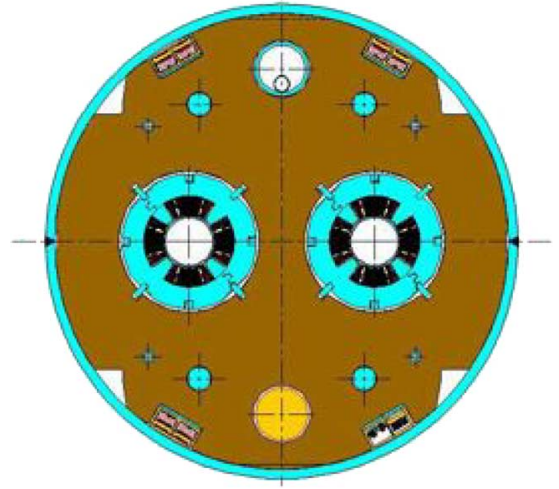

Fig. 8. LHC quadrupole for the matching sections.

The rest, i.e. the vast majority, are operated in superfluid helium like the main dipoles. All quadrupoles are of the two-in-one type, i.e. the coils for the two-beam channel are in the same iron yoke, while each coil is collared as single unit. The collared coil is a freestanding unit in the iron yoke, i.e., collars support all the forces, see Fig. 8. This is possible because the forces, like the stored energy are noticeably less for a quadrupole than for similar bore dipoles. Moreover, each channel is magnetically decoupled by means of an iron central leg between the two coils, see again Fig. 8. These quadrupoles are much shorter than the dipoles, ranging from 3 to $4.5 \mathrm{~m}$. However, the cold masses range from $7.5 \mathrm{~m}$ of the arc SSS to $15 \mathrm{~m}$ for some S4 where two main quadrupoles or many large correctors are assembled in one S4. The complication given by the number of variant of the SSS and S4 cold masses make any repair or substitution much more difficult than in the case of the dipoles.

A further family of important quadrupoles consists of the interaction regions (IR) quadrupoles. These are the 32 quadrupoles that have to provide the strong focusing of the beams at the interaction points. Indeed, the more beam is squeezed the higher is the particle density and the more frequent are the collisions. The magnets are the ultimate determinant of the Luminosity, a parameter defined as the collision rate times the cross section and that, after the energy, is the most significant measure for collider performance. These quadrupoles are single aperture magnets with a coil bore of $70 \mathrm{~mm}$ (versus $56 \mathrm{~mm}$ for the main dipoles and quadrupoles). Because of the high gradient and large aperture the peak field in these quadrupoles is near the peak field in the dipole. Operation at $1.9 \mathrm{~K}$ is required both by the peak field value and by heat removal: indeed because these magnets gets a considerable part of the collision debris escaping from detectors along the beam tube, they have to be stable against continuous energy release of $3 \mathrm{~mW} / \mathrm{cm}^{3}$, which means $30 \mathrm{~W}$ per magnet. These special IR quadrupoles, together with the 16 associated superconducting dipoles for beam merging and separation, are a special contribution of the US DOE labs (Fermilab, BNL and LBNL) [28] and of Japan (KEK) [29].

\section{CERN Supply: Components, Large Tooling and Magnetic Measurements}

As part of a cost saving strategy and in order to keep under control the characteristics of the magnet components having some impact on the final quality and on the schedule, all main 


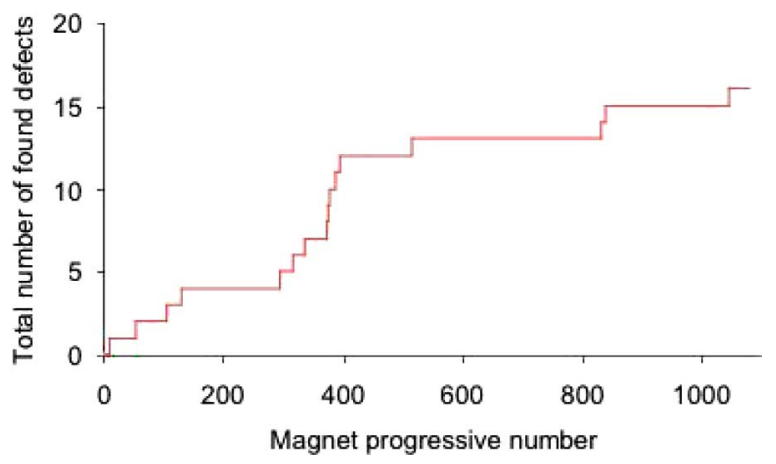

Fig. 9. Defects detected through magnet measurements during production on the main dipole coils.

components are supplied by CERN to the magnet manufacturers, including the precious superconducting cables, in single unit lengths, sorted for similar properties for each magnet. This strategy implies that CERN became supplier of its suppliers with an intricate share of responsibility that made CERN fully responsible for the magnet performance, except in case of negligence or clear fault of the dipole manufacturer.

Some of the main tooling for the dipole production, namely the large presses used for coil collaring and for cold mass welding were specified and procured directly by CERN. In order to meet the technical specification of the welding of the shrinking cylinder and to provide a reliable and fast welding procedure, after considerable $\mathrm{R} \& \mathrm{D}$, it was chosen to have the root pass welded by STT (speed: $70 \mathrm{~mm} /$ minute) and then to fill the seam with three passes of MIG $(200 \mathrm{~mm} / \mathrm{min}$.). STT is a rather new process, used in industry for only a few years and not yet industrialized for automatic welding on austenitic steel.

Another special tooling procured by CERN is a Laser Tracker that is fundamental for measuring the curvature, planarity, twist, and inter-aperture distance of the magnets, all along the $15 \mathrm{~m}$ long narrow Cold Bore Tube (CBT), with the specified $0.1 \mathrm{~mm}$ precision. It is also essential to measure the position (and actually to facilitate in the positioning during their assembly), of all the "3-D" components, i.e. at the magnet extremities.

Special equipment was also provided by CERN for magnetic measurements at warm (twelve systems in total). By performing measurements and analysis at various stages of the manufacture [30], a few errors were detected as shown in Fig. 9. All defects could be repaired, by disassembly of the collared coils and procedures were improved.

\section{Performance}

The magnets are delivered as cold masses. At CERN the following tasks take place: i) cryostat assembly; ii) cold test (power and magnetic field measurements); iii) preparation for the tunnel with insertion of a beam screen to intercept the synchrotron radiation (that otherwise would constitute an unacceptable load on the $1.9 \mathrm{~K}$ cryogenics); iv) transport in the tunnel; v) interconnections between magnets. Eventually cool down and energization in the tunnel can start, an operation that is foreseen in winter 2007 for the first octant and November 2007 for the last one.

Cold testing of all individual magnets has allowed intercepting a few magnets with weak quench performance or

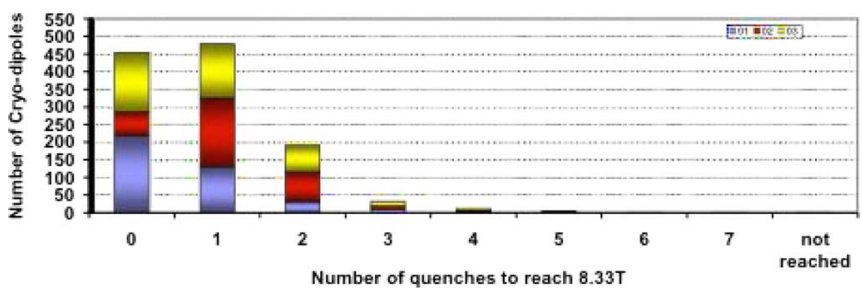

Fig. 10. LHC dipole quench performance at first thermal cycle.

showing electrical defaults. In Fig. 10 the quench performance at first thermal cycle of the dipoles tested so far is shown [31]. Magnets reaching nominal operation in two or less training quenches (nearly $90 \%$ so far) are considered very good. Some of the others are submitted to a new thermal cycle and then re-tested. After more than 1000 dipoles tested only $2.5 \%$ were found defective: 11 for electrical problems (mainly quench heater damage) and 14 for quench performance. Almost all have been repaired and returned to CERN.

About $10 \%$ of the dipoles have been fully measured to assess the field quality content. By means of well-established correlation between magnetic fields at cold and warm the harmonic content in operation coming from the coil geometry is statistically well known on all magnets. Measurements in superconducting state have allowed the evaluation of the field errors coming from persistent currents and snap back, which are of great importance for the initial phase of the acceleration process [32].

\section{DETECTORS}

We mentioned that detector magnets are usually of large size and moderate field. However in the LHC the size is extremely large, $25 \mathrm{~m}$ long for the ATLAS Barrel Toroid (BT), and the field is not so low, $4 \mathrm{~T}$ in the center of the $12 \mathrm{~m} \mathrm{CMS} \mathrm{solenoid.} \mathrm{So}$ the challenge is quite serious. A further characteristic typical of detector magnets is that they have to be integrated in a complex object and the room for coil, its cryostat and the mechanical structure is frequently very scarce: basically physicists would like a magnet-less field. Magnet size and consequent stored energy (CMS being the champion of SC magnets with its 2.7 GJ of stored energy), implies that superconducting design of detector magnets is dictated by two factors: 1) magnet protection, since in any case and whatever scenario the risk of loosing the magnet cannot be tolerated because of the cost and of the fact that the whole detector is built around the magnet; 2) magnet stability, since, given the dimensions, perturbation are large and if a magnet quenches, it will mean loss of a few weeks of detector data taking.

\section{A. Conductor Generalities}

Both ATLAS and CMS magnets are wound with $\mathrm{Cu} / \mathrm{Nb}-\mathrm{Ti}$ flat Rutherford cables embedded through co-extrusion in a pure aluminum matrix, a technology developed and well established for detector magnets. Bonding values between cables and aluminum above 15-20 MPa are required. To assure this value over the $100 \mathrm{~km}$ long production needed for the two experiments, an extremely careful QA plan and tooling was set up. A new 


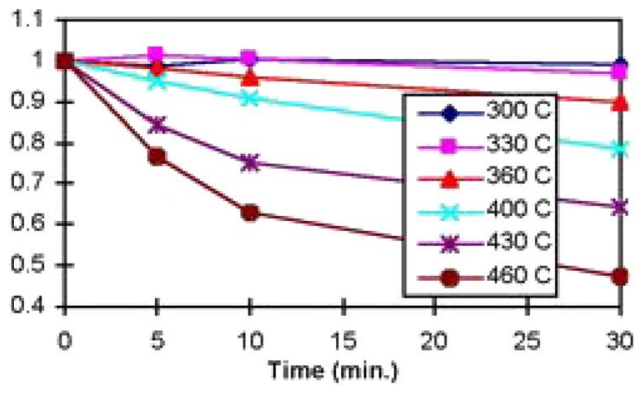

Fig. 11. Critical current degradation of $\mathrm{Cu} / \mathrm{Nb}$-Ti versus thermal treatment simulating co-extrusion conditions.

device, based on an ultra-sound scanner that is able to continuously monitor the bonding by means of imaging analysis has been developed. Co-extrusion parameters have been carefully studied during the R\&D phase, to avoid a severe reduction of the critical current: the goal is usually to limit Ic degradation to less than $10 \%$ from virgin wire. Given $2 \%-4 \%$ of degradation for cabling, this allows only $6 \%-8 \%$ degradation for co-extrusion. Since temperature can vary between 390 and $420^{\circ} \mathrm{C}$ for a time of 30-100 s, according to the process and the speed used, one can see from Fig. 11 that this goal is at the limit of present technology.

A very good RRR of the aluminum can be achieved by using commercial-expensive-Aluminum $99.998 \%$, (RRR greater than 2500 in the billet) and by carefully controlling the cleaning of the press. Values of 1200 and more have been achieved in ATLAS and CMS finished conductor.

A further parameter that has to be optimized, is the contact resistance between cable and aluminum matrix. Indeed the bonding is given by the copper-aluminum inter-metallic layer, typically 1-2 $\mu \mathrm{m}$ thick. Lack of the inter-metallic layer generates a region of bad bonding, and even bad electrical contact between cable and stabilizer. But a too thick inter-metallic barrier, whose resistivity is pretty high, can increase the contact resistance above optimal values, thus reducing the stability (see later in the paper). For ATLAS and CMS an inter-metallic thickness of less than $3 \mu \mathrm{m}$ assures contact resistance of less than $10-11 \mathrm{n} \Omega \mathrm{m}$ at zero field, which has been proved to be largely sufficient for stability in the B0 model coil test.

Generally speaking, indirect cooling coupled with aluminum stabilized conductor is the most economic solution for large systems: for example the ATLAS toroid conductor costs less than 2 Euros/kA-m @ 5 T, $4.2 \mathrm{~K}$ and the cost of the Atlas magnet system (one of the largest and most complex superconducting magnet systems) is less than $90 \mathrm{M}$ Euros.

Indirect cooling and large current, $20 \mathrm{kA}$, call for low resistance of the joints between conductors, less than $0.5 \mathrm{n}$ ? in magnetic field. One further problem of these junctions is that conductors are so big that in some cases the flux linked to the junction is not negligible, and the induced flux can quench the magnet, especially in ramping down. This effect usually requires short junction length, while good contact resistance calls for junction as long as possible (since a resistive barrier, the aluminum, is present). For ATLAS an accurate study confirmed by experimental results has fixed the optimal length to be around $2.5 \mathrm{~m}$.

\section{B. Stability and Protection}

1) Stability and Margin: Cooling capacity is large and supercritical helium flowing in the channel can remove from hundreds of watts to tens of $\mathrm{kW}$. But there is considerable thermal resistance between the coils, where heat is generated, and the cooling channels where heat is eventually removed. So there has to be a fairly good enthalpy margin. For ATLAS BT, for example, the choice has been $\Delta \mathrm{H} \approx 2 \mathrm{~kJ} / \mathrm{m}^{3}$, that is accomplished by a temperature margin $\Delta \mathrm{T} \mathrm{E} 6.8-4.8=2 \mathrm{~K}$. This margin, together with the good conductivity of the matrix, can also cope with an internal joint with moderately high resistance.

Given the amount of stabilizing aluminum in the conductor cross section, $400-600 \mathrm{~mm}^{2}$, the MPZ (Minimum Propagating Zone) length is about $1 \mathrm{~m}$ for ATLAS toroids and CMS solenoid and the MQE (Minimum Quench Energy) against point disturbances is about $5 \mathrm{~J}$.

Both enthalpy margin and transient effect stability call for a large margin in $\mathrm{I}_{\mathrm{c}}$. Typically a detector magnet works at $35 \%-40 \%$ of its $\mathrm{I}_{\mathrm{c}}$. That translates to a working point at about $60 \%-65 \%$ of the maximum current along the load line. Given the margin and the fact that the conductor cross-section is dominated by the matrix, $\mathrm{J}_{\mathrm{c}}$ is never an issue because it can be compensated largely by an increase in $\mathrm{Nb}$-Ti cross section, without prejudice for the stabilizer content.

2) Protection: The amount of stabilizing aluminum is eventually determined by protection consideration. Indeed we need to buy time to safely detect (and with certainty, to avoid useless fast discharge) the quench onset in such a large coil. Further more time is needed to extract energy and/or to spread energy into the coils to avoid dangerous hot spots, too high thermal stresses and unsustainable voltages. The main reasons for large conductors can therefore be summarized as: large current, in order to have low inductance, and large stabilizing cross section to lessen the $\mathrm{J}_{\text {stab }}$ after transition.

All magnet systems of ATLAS rely on heaters. In all cases, it is necessary to spread out the quench rapidly. In the case of the toroids, since the quench margin is so huge, $\mathrm{kW}$ of heater power has been installed and accurate studies to select their positions have been carried out. A very effective technique to spread out a quench, used successfully in the Atlas inner solenoid, is to bond longitudinal strips of pure aluminum over the coil ground insulation, using them as passively activated quench heaters. In case of CMS a more classical system based mainly on an external dumping resistor has successfully been installed. In this case the cold mass rise in temperature is less than in the case of spreading the quench inside: the price to pay is however a larger voltage across the coil terminals, about $1 \mathrm{kV}$.

Protection and stabilization both demand a matrix having very high conductivity. This is "easily" accomplished by using pure aluminum, as previously mentioned. It is worth noting that the RRR $=1000$ at zero field becomes 400 at $1 \mathrm{~T}$ and for higher field the $\mathrm{Al}$ magneto-resistivity saturates, which is another advantage of pure aluminum over copper.

To conclude this overview of protection, it is instructive to consider the graph, see Fig. 12, where most of the main solenoidal detector magnets built so far are represented in a plot of Energy/Mass vs. Energy. The plot should be complemented by other information: for examples thin solenoids like ZEUS 


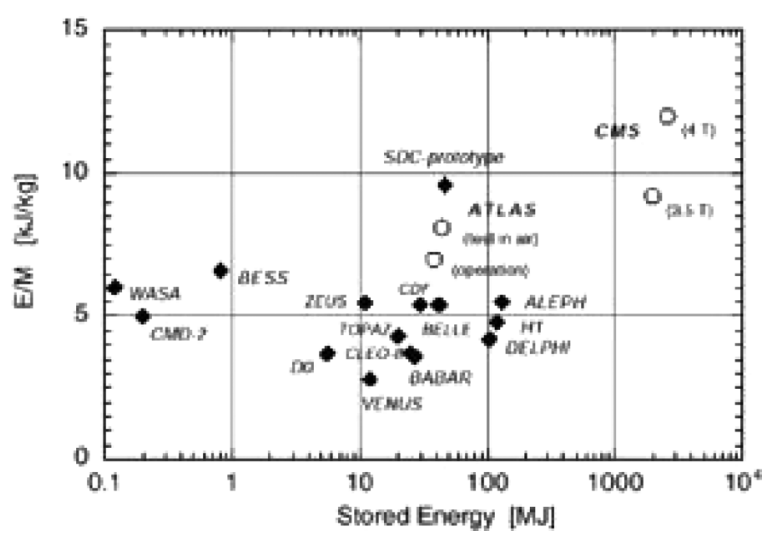

Fig. 12. Stored energy per unit cold mass versus stored energy for many HEP detector magnets.

and ATLAS have more and tighter constraints than the muon chamber magnets like Delphi, Aleph and CMS. However it shows the great jump required by the LHC detectors. A review of detector magnets, oriented towards giant system like the LHC detectors, can be found in [34], [35].

\section{General Lay-Out and Mechanical Structure}

Two main structures are employed for detectors: solenoid and toroid. Most magnets are solenoids, having the advantage of a good efficiency factor: $\Gamma=\mathrm{B}_{a v} / \mathrm{B}_{p e a k}$, where $\mathrm{B}_{a v}$ is the average field seen by particles in their trajectories and $\mathrm{B}_{\text {peak }}$ is the peak field on the coil. Indeed for the CMS and Atlas solenoids $\Gamma$ is of the order of 0.85-0.9, CMS having a $\mathrm{B}_{\text {peak }}$ of $4.6 \mathrm{~T}$, while for the ATLAS toroids it is of course much worse $\Gamma \approx 0.25$ (for $\mathrm{a} \mathrm{B}_{\text {peak }}=3.8 \mathrm{~T}$ ). But the toroid configuration has the advantage of field lines always perpendicular to the particle trajectories, so the bending strength for forward particles is higher for toroids than for solenoids.

As far as force containment is concerned, it should be noted that pure aluminum has very poor mechanical characteristics and this determines the mechanical structure.

In case of a toroid, like ATLAS, the coils are fully impregnated and constrained in coil casings. These casings are made of aluminum alloy for considerations of weight and for compatibility with coil thermal shrinkage (coils are dominated by aluminum properties). Coil casings are held one against the other by a castellation of struts (see Fig. 13). Of course the lack of any self-support for toroidal coils, like for accelerator magnets, makes the force and stress modeling and computing very critical.

Another possibility is the inner winding technique, which has been used for the CMS solenoid. Here the coil is wound onto the cylinder from the inner side. This technique is widely used and applies very well when cylinders are larger than $2 \mathrm{~m}$ in diameter. However for CMS the coil package is so thick that stresses must be intercepted at each of the four conductor layers. The CMS conductor has been reinforced by means of two strips of special aluminum alloy that are bonded to the pure aluminum matrix by means of a suitably developed e-beam welding line. This gives a rigidity to the conductor that, while it participates in stress containment, has made winding on the inner side especially difficult, necessitating very special tooling.

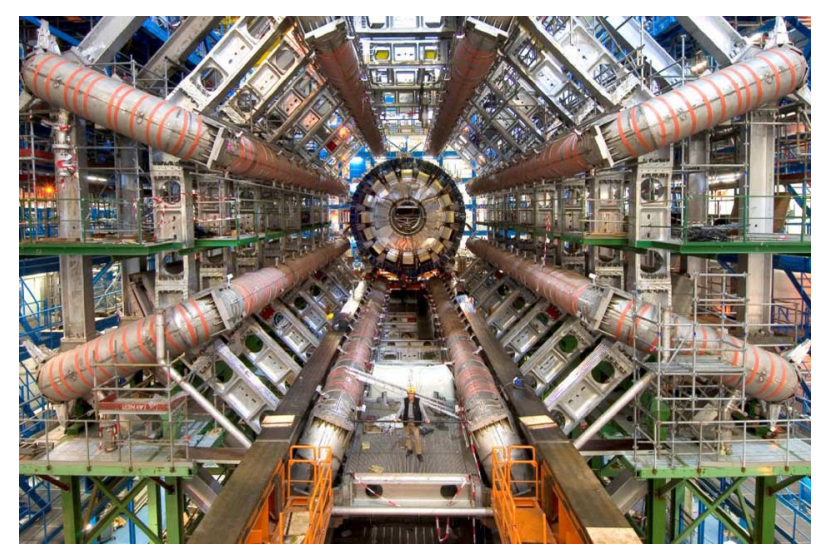

Fig. 13. The ATLAS BT magnet after installation in the cavern.

\section{ATLAS: The Giant Toroid}

Effectively, because of a certain interest of having the whole detector to be practically iron-free, to avoid multiple scattering limitations in the devices that detect the particles, the toroidal configuration is more and more considered for detectors. The ATLAS collaboration, 1800 physicists and engineers from 164 Universities and Institutes from 35 countries, has designed and built a complex system composed by four large magnets.

The first is a large ( $25 \mathrm{~m}$ long, $5 \mathrm{~m}$ wide) toroid covering the whole length of the detector, called barrel toroid (BT), see Fig. 13. The toroid is composed of 8 coils and it serves as main mechanical structure of the detector.

Two end cap toroids (ECT) close the barrel toroid and take care of bending the forward particles. ECT and BT are powered in series and share the same protection system and cryogenic system.

The inner solenoid is $2.4 \mathrm{~m}$ in diameter and $5.3 \mathrm{~m}$ in length. By generating a field of $2 \mathrm{~T}$ it provides an optimized bending for particles inside the trackers. In order to minimize the room allocated for mechanical structure a special aluminum alloy has been developed. It shows a modest decrease in RRR while the yield strength it is increased to $130 \mathrm{MPa}$. This allows the aluminum, whose presence is necessary for protection and stability, to be used also for force containment.

At present the solenoid has been fully tested in its final position [36], while the BT, after successful testing of all 8 coils at $110 \%$ of operating current, will be tested in the detector in autumn 2006 [37]. The ECT test is foreseen in summer 2007 [38].

\section{E. CMS: The Most Powerful Solenoid}

CMS, designed and built by a collaboration of the similar size as ATLAS, employs a more classical configuration, already exploited in large detectors like Aleph and Delphi: a large solenoid whose coil is placed outside the calorimetric part. Given the size, $7 \mathrm{~m}$ in diameter and $13 \mathrm{~m}$ in length, and the $4 \mathrm{~T}$ field level, the stored energy is exceptional: 2.7 GJ. Powered up to nominal field first on August 29, 2006 [39], it holds, presumably for long time, the world record as steady magnetic energy storage. The hoop forces are so high that, despite a $50 \mathrm{~mm}$ thick restrain cylinder, made out of strong $\mathrm{Al}$ alloy, the conductor must have a special reinforcement, as previously mentioned. The winding has to assure good field uniformity despite the rigidity of the 


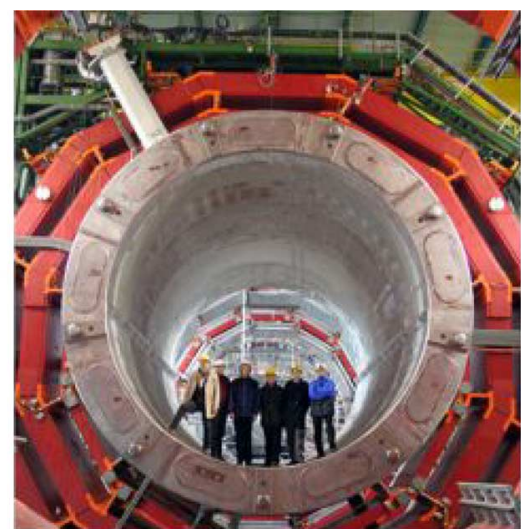

January 2006: End of the CMS Magnet Manufacturing

Fig. 14. CMS solenoid inside the dodecagonal iron yoke.

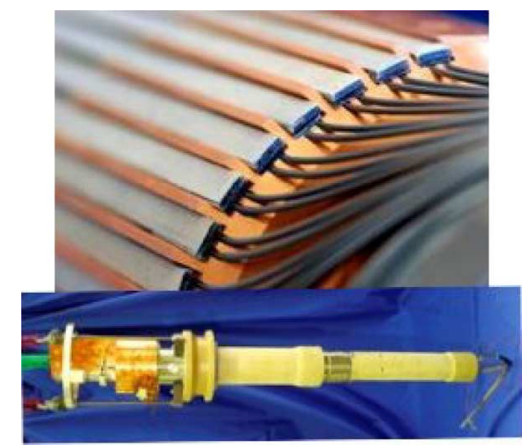

Fig. 15. 13 kA HTS current lead. Stacks brazed to the main body (above) and finished lead (below).

conductor: the coil has been wound from the inside onto the restraining cylinder by pre-bending the cable and positioning it by means of a robot.

In Fig. 14, the CMS magnet is shown before first excitation, on the surface.

\section{HTS CURRENT LEADS IN THE LHC}

To power the numerous LHC superconducting magnets of the accelerator, about $3 \mathrm{MA}$ of electrical current need to be fed into the cold circuits, at various current ratings and locations. To save heat load at cryogenic temperatures, the current leads rated at currents higher than $120 \mathrm{~A}$ are based on Bi-2223 tapes, stabilized with an Ag-Au alloy to cut down the thermal conduction into the He bath. The design of the leads makes use of available He gas at 5-20 K, which absorbs the heat dissipated in the resistive upper section, operating from room temperature down to $50 \mathrm{~K}$.

From $50 \mathrm{~K}$ down to $4.5 \mathrm{~K}$, stacks of $\mathrm{Bi}-2223$ tapes, soldered at their cold end to $\mathrm{Nb}$ - $\mathrm{Ti}$ wires, carry the current to the $\mathrm{Nb}-\mathrm{Ti}$ bus-bars that deliver power to the superconducting magnets. In total 1030 HTS current leads have been manufactured, including the 32 pairs rated at $13 \mathrm{kA}$, shown in Fig. 15. In total, $31 \mathrm{~km}$ of Bi-2223 tape has been successfully manufactured by AMSC and EHTS and then assembled, at CERN, into more than 10000 stacks. All these stacks are individually tested at liquid nitrogen temperature, before being brazed in the main body of the lead [40].

\section{CONCLUSIONS AND ACKNOWLEDGEMENTS}

The LHC is one of the largest scientific enterprises and the largest application of superconducting and cryogenic technologies. Its construction relies on custom design and on manufacturing technologies that, although difficult, can be considered industrially matured. Superconductivity, both LTS and HTS, each one in its domain of application, is proving to be an enabling technology for large-scale equipment.

More than 100 scientists and engineers are dealing with the superconducting part of this project since more than ten years, and many others have collaborated on the industry side. The success of LHC should be credited first to all these people of the superconductivity and cryogenic community that have worked so hard to make possible such a huge project.

I would like recognize the contribution of the numerous students and young fellows involved in the studies, R\&D and then construction of the project: LHC is based also on their work and dedication.

\section{REFERENCES}

[1] M. N. Wilson, "Superconductivity and accelerators: The good companions," IEEE Trans. Appl. Supercond, vol. 9, pp. 111-121, 1999.

[2] K.-H. Mess, P. Schmüser, and S. Wolff, Superconducting Accelerator Magnets. Singapore: World Scientific, 1996.

[3] F. M. Asner, High Field Superconducting Magnets. Oxford: Clarendon Press, 1999

[4] A. Devred, "Superconducting magnets for particle accelerators and storage rings," Wiley Encyclopedia of Electrical and Electronics Engineering, vol. 20, pp. 743-762, 1999.

[5] L. Rossi, "Superconducting magnets for accelerators," in Superconducting Materials for High Energy Colliders, L. Cifarelli and L. Maritato, Eds. Singapore: World Scientific, 2001, pp. 106-122.

[6] J. R. Purcell, "The 1.8 tesla, $4.8 \mathrm{~m}$ I.D. bubble chamber magnet," in Proceedings of the 1968 Summer Study on Superconducting Devices and Accelerators, Brookhaven National Laboratory June 10-July 19, 1968, pp. 765-785.

[7] K. Jaeger and J. Purcell, "Operation and accuracy of the 12-foot bubble chamber magnet," in Proc. 4th Int. Conf. on Magnet Technology (MT4) Brookhaven, 1972, pp. 328-338.

[8] A. V. Tollestrup, "Superconducting magnets," in Physics of High Energy Accelerators, R. A. Carrigan, F. R. Hudson, and M. Months, Eds. : AIP Proceedings, 1979, vol. 87, pp. 699-804.

[9] H. T. Edwards, "The Tevatron energy doubler: A superconducting accelerator," Annual Review of Nucl. Part. Sci., vol. 35, pp. 605-660, 1985.

[10] LHC Design Report, “The LHC Main Ring,” CERN-2004-003, June 2004, vol. I.

[11] S. Wolff, "Superconducting HERA magnets," IEEE Trans. Magn., vol. 24 , no. 2, pp. 719-722.

[12] M. Anerella et al., "The RHIC magnet system," Nucl. Instrum. Meth., vol. A499, pp. 280-315, 2003.

[13] ATLAS Collaboration, "ATLAS Technical Design Report, Magnet System," 4 Volumes CERN/LHCC/97-18,19,20,21, 1997.

[14] CMS collaboration, "Magnet Project: Technical Design Report," CERN/LHCC/97-10, 1997

[15] P. Lebrun, "Superconductivity and cryogenics for future high-energy accelerators," presented at the ICEC21 Conference, submitted for publication of the proceedings.

[16] R. D. Heuer, "The International Linear Collider ILC-A status report," Nuclear Physics B-Proceedings Supplements, vol. 154, no. 1, pp. 131-136, April 2006

[17] L. Rossi, "State-of-the-art accelerator magnets," IEEE Trans. Appl. Supercond., vol. 12, no. 1, pp. 219-227, March 2002.

[18] A. Asner, R. Perin, W. Wenger, and F. Zerobin, "First Nb3Sn superconducting dipole model magnets for the LHC break the 10 tesla field threshold," in Proc. of MT-11 Conference, Tsukuba 1989, Elsevier Applied Science, 1990, pp. 36-41.

[19] R. Perin, "The superconducting magnet system for the LHC," IEEE Trans. Appl. Supercond, vol. 3, 1991.

[20] D. Leroy, "Review of the R\&D and supply of the LHC superconducting cables," IEEE Trans. Appl. Supercond., vol. 16, pp. 1152-1159, June 2006. 
[21] A. Verweij, "Critical current measurements of the main LHC superconducting cables," IEEE Trans. Appl. Supercond., vol. 17, 2006, submitted for publication.

[22] L. Rossi, "Superconducting magnets for the LHC main lattice," IEEE Trans. Appl. Supercond, vol. 14, pp. 153-158, June 2004.

[23] E. Todesco et al., "Steering field quality in the main dipole magnets of the large hadron collider," IEEE Trans. Appl. Supercond., vol. 14, pp. 177-180, June 2004.

[24] M. Bajko, P. Fessia, and D. Perini, "Statistical studies of the robustness of the LHC main dipole mechanical structure," IEEE Trans. Appl. Supercond., vol. 10, no. 1, pp. 77-80, March 2000.

[25] F. Bertinelli, F. Fudanoki, T. Komori, G. Peiro, and L. Rossi, "Production of austenitic steel for the LHC superconducting dipole magnets," IEEE Trans. Appl. Supercond., vol. 16, pp. 1773-1776, June 2006.

[26] R. Burgmer et al., "Industrialization of LHC main quadrupoles cold masses up to series production," IEEE Trans. Appl. Supercond., vol. 14, pp. 169-172, June 2004.

[27] R. Ostojic, "Superconducting magnets for the LHC insertions," IEEE Trans. Appl. Supercond., vol. 14, pp. 181-186, June 2004.

[28] R. Bossert et al., "Test results from the LQXB quadrupole production program at fermilab for the LHC interaction regions," IEEE Trans. Appl. Supercond., vol. 14, pp. 187-190, June 2004.

[29] Y. Ajima et al., "The MQXA quadrupoles for the LHC low-beta insertions," Nucl. Inst, and Meth. in Phys. Res. A, vol. 550, pp. 499-513, April 2005.

[30] C. Vollinger and L. Todesco, "Identification of assembly faults through the detections of magnetic field anomalies in the production of the LHC main dipoles," IEEE Trans. Appl. Supercond., vol. 16, pp. 204-207, June 2006.
[31] P. Pugnat and A. Siemko, "Review of quench performance of the LHC main superconducting magnets," IEEE Trans. Appl. Supercond., vol. 17, 2006, submitted for publication.

[32] S. Amet, L. Bottura, V. Granata, S. Le Naour, R. Mishra, L. Oberli, D. Richter, S. Sanfilippo, A. Verweij, L. Walckiers, and R. Wolf, "Persistent and coupling current effects in the LHC superconducting dipoles," IEEE Trans. Appl. Supercond., vol. 13, pp. 1239-1242, 2003.

[33] P. Miele et al., "ATLAS B0 toroid model coil test at CERN," IEEE Trans. Appl. Supercond., vol. 12, pp. 411-415, June 2002.

[34] A. Yamamoto et al., "Development towards ultra-thin superconducting solenoid magnets for high energy particle detectors," Nuclear Physics B-Proceedings Supplement, vol. 78, no. 1-3, pp. 565-570, August 1999.

[35] L. Rossi, "Superconducting magnets for accelerators and detectors," Cryogenics, vol. 43, pp. 281-301, 2003.

[36] R. J. M. Y. Ruber et al., "Ultimate performance of the atlas superconducting solenoid," IEEE Trans. Appl. Supercond, vol. 17, submitted for publication.

[37] H. H. J. ten Kate, "ATLAS superconducting magnet system performance," IEEE Trans. Appl. Supercond., vol. 17, on behalf of the Atlas Collaboration, submitted for publication.

[38] D. E. Baynham et al., "ATLAS end cap toroidal integration and test," IEEE Trans. Appl. Supercond., vol. 17, submitted for publication.

[39] D. E. Campi et al., "Commissioning of the CMS magnet," IEEE Trans. Appl. Supercond., vol. 17, submitted for publication.

[40] A. Ballarino, L. Martini, S. Mathot, T. Taylor, and R. Brambilla, "Large scale assembly and characterization of Bi-2223 conductors," IEEE Trans. Appl. Supercond., vol. 17, submitted for publication. 